\title{
Cancer Treatment-Induced Bone Loss and Role of Denosumab in Nonmetastatic Prostate Cancer: A Narrative Review
}

\author{
Deepak Dabkara ${ }^{1}$ \\ ${ }^{1}$ Department of Medical Oncology, Tata Medical Center, Kolkata, \\ West Bengal, India
}

Ind J Med Paediatr Oncol 2021;42:240-246.
Address for correspondence Deepak Dabkara, MD, DM, Department of Medical Oncology, Tata Medical Center, Kolkata 700136, West Bengal, India (e-mail: deepakdabkara@ yahoo.com).

\begin{abstract}
Keywords

- nonmetastatic prostate cancer

- denosumab

- bone loss

- bone mineral density

- fracture

Bone loss is an important complication of prostate cancer and its associated treatments, especially androgen-deprivation therapy (ADT). There is a 5 to 10 times increased loss of bone mineral density (BMD) in men receiving ADT with yearly 4 to $13 \%$ BMD loss. The risk of fracture increases yearly by 5 to $8 \%$ with ADT. ADT associated bone loss of 10 to $15 \%$ of BMD doubles the risk of fractures. Hence, BMD evaluation through dual-energy $\mathrm{X}$-ray absorptiometry and evaluation of individual fracture risk assessed before initiating ADT. The use of vitamin D, calcium, bisphosphonates, and denosumab has shown improved bone health in men with prostate cancer receiving ADT. Denosumab $60 \mathrm{mg}$ is approved to increase bone mass in men at high risk for fractures receiving ADT for nonmetastatic prostate cancer. Denosumab has shown improvement of $5.6 \%$ BMD at 2 years in nonmetastatic prostate cancer patients, with significant improvements seen at the total hip, femoral neck, and distal third of the radius. Denosumab has shown a $62 \%$ decreased incidence of new vertebral fractures at 36 months. Furthermore, denosumab delays the onset of bone metastases in high-risk nonmetastatic prostate cancer patients. Denosumab can be preferred over other bone modifying agents owing to several advantages, such as subcutaneous administration and no requirement of hospitalization, no dose modifications in renal impairment and less incidence of acute phase anaphylactic reactions. We review the available evidence of denosumab for managing bone loss in nonmetastatic prostate cancer patients. The relevant articles used in this narrative review were obtained through general search on google and PubMed using the key terms "non-metastatic prostate cancer," "denosumab," "bone loss," "bone mineral density," "fracture," "CTIBL," and "chemotherapy induced bone loss."
\end{abstract}

\section{Key Clinical Takeaways}

- Dual-energy X-ray absorptiometry evaluation for bone mineral density (BMD) and evaluation of individual fracture risk should be performed prior to starting androgen-deprivation therapy (ADT).

DOI https://doi.org/

$10.1055 / \mathrm{s}-0041-1732851$

ISSN 0971-5851
- Denosumab (60 mg at every 6 months given subcutaneously) is recommended to increase bone mass in men who are at a high risk of fracture from receiving ADT for nonmetastatic prostate cancer.

- Denosumab can be preferred over bisphosphonates, as it does not require renal monitoring and dose modification

(C) 2021. Indian Society of Medical and Paediatric Oncology.

This is an open access article published by Thieme under the terms of the Creative Commons Attribution-NonDerivative-NonCommercial-License, permitting copying and reproduction so long as the original work is given appropriate credit. Contents may not be used for commercial purposes, or adapted, remixed, transformed or built upon. (https://creativecommons.org/licenses/by-nc-nd/4.0/).

Thieme Medical and Scientific Publishers Private Ltd. A-12, Second Floor, Sector -2, NOIDA -201301, India 
in renally impaired patients with a better safety profile. It can be easily administered (subcutaneous) by patients themselves.

- Cost of biosimilar denosumab is much lower than the innovator denosumab which might benefit a larger proportion of prostate cancer patients.

- Denosumab is not recommended in patients with hypocalcemia.

- A regular monitoring of calcium and minerals (magnesium and phosphorus) within 14 days is required in patients receiving denosumab due to risk of worsening of hypocalcemia.

- Serial dental examination with appropriate preventive dentistry is recommended due to the risk of osteonecrosis of the jaw.

- In view of the increased risk of fractures after denosumab stoppage, a rapid transition to alternative antiresorptive treatment should be considered.

\section{Introduction}

According to the GLOBOCAN 2018 data, prostate cancer $(1,276,106$ new cases; $7.1 \%$ of all cancer cases) is the second commonest cancer in men globally and has the seventh highest cancer-related mortality (358,989 deaths, 3.8\% of all cases). ${ }^{1}$ The management of prostate cancer has been traditionally with androgen-deprivation therapy (ADT, including bilateral orchiectomy or medical castration) using gonadotropin-releasing hormone $(\mathrm{GnRH})$ agents. $^{2}$ Approximately half of the prostate cancer patients receive ADT at some point after diagnosis. ${ }^{3,4}$ Cancer therapy leads to osteopenia and osteoporosis which are termed as cancer treatment-induced bone loss (CTIBL). We review the impact of CTIBL in nonmetastatic prostate cancer patients and the role of denosumab in improving bone mass in such patients.

\section{Bone Loss in Prostate Cancer}

Majority of the prostate cancer patients (>70\%) are elderly ( $\geq 65$ years) who are already associated with a high risk for osteoporosis. ${ }^{5}$ Furthermore, cancer therapy also leads to osteopenia and osteoporosis which are termed as CTIBL. ${ }^{6.7}$ In prostate cancer patients, testosterone deficiency is seen secondary to androgen deprivation from ADT including GnRH agents or surgical castration. ${ }^{7.8} \mathrm{ADT}$ is known to induce bone loss, thereby increasing the risk of osteoporosis and bone fractures, ultimately affecting the quality of life (QOL) of patients. ${ }^{6}$

\section{Pathophysiology}

The binding of ligand receptor activator of nuclear factor-кB (RANK) to its ligand RANKL triggers a signal transduction pathway which subsequently stimulates osteoclast functioning, whereas osteoprotegerin binds to RANKL preventing bone resorption. ${ }^{9-11}$ The binding of RANK to RANKL causes osteoclast differentiation in the presence of colony stimulating factor 1 (CSF1), and bone resorbing activity in mature osteoclasts. Osteoprotegerin after binding to RANKL inhibits osteoclastogenesis and osteoclast activation ( - Fig. 1). ${ }^{12}$

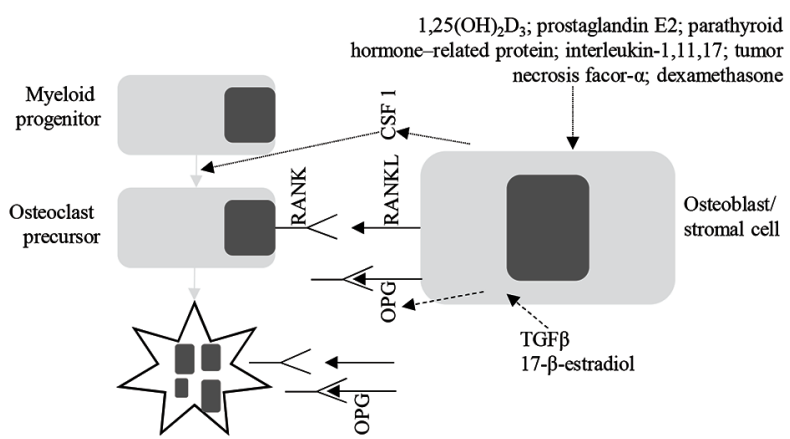

Fig. 1 Regulation of osteoclast formation. Adapted from: Jones et al. ${ }^{12}$ CSF1, colony stimulating factor 1; RANK, receptor activator of nuclear factor-KB; RANKL, RANK ligand; OPG, osteoprotegerin; TGF, transforming growth factor.

\section{Cancer Treatment-Induced Bone Loss}

Decrease in estrogen and testosterone occurring with hypogonadism leads to faster bone loss, and in the absence of increased bone formation, it may lead to osteopenia and eventually osteoporosis. ${ }^{8}$ In men with prostate cancer receiving $\mathrm{ADT}$, the decreased circulating testosterone and estrogen, decreased osteoblastic bone formation, and increased osteoclastic bone resorption result in accelerated bone loss. ${ }^{13,14}$ There could be a decrease in muscle mass due to ADT-induced hypogonadism, which may decrease the mechanical stretch and pressure leading to loss in the bone mineral density (BMD)..$^{15}$ Glucocorticoids including dexamethasone, which are generally given along with some chemotherapeutic agents for the treatment of prostate cancer, are also known to increase the risk of secondary osteoporosis and fractures. ${ }^{16,17}$

\section{Androgen-Deprivation Therapy and Bone Loss}

The ADT-induced treatment causes decrease in testosterone and estrogen, resulting in an accelerated and disorganized bone turnover. ${ }^{18,19}$ ADT is associated with significant bone loss and increased risk of fractures. ${ }^{19,20}$ There is a 5- to 10-time increased BMD loss with ADT usage. ${ }^{21}$ The decrease in BMD is maximum in the first year of ADT therapy (up to $4 \%$ ), and continuation of use is associated with ongoing loss in subsequent years along with the increased risk of fractures. ${ }^{21-25}$

Duration of Androgen-Deprivation Therapy and Bone Loss: Decrease in Bone Mineral Density and Risk of Fracture The duration of ADT affects the decrease in BMD, prevalence of osteoporosis, and risk of fractures. ${ }^{26,27}$ A significant BMD loss at 6 to 12 months is seen in patients receiving continuous ADT ( $4-13 \%$ per year); up to $10 \%$ bone loss is seen following 2 years of $A D T, 5,28$ In the first year during ADT, the loss of bone mass is most pronounced, which subsequently continues at a slower pace beyond the second year. ${ }^{11,21}$ Morote and colleagues reported a similar decrease in BMD from baseline throughout the ADT treatment with most pronounced decrease in the initial years followed by a slower decrease throughout. Osteoporosis rates increased from 35.4 to $42.9 \%$ after 2 years of ADT, which eventually increased to $80.6 \%$ after $\geq 10$ years. ${ }^{29}$ 
The risk of fractures also increases at an average 5 to $8 \%$ per year with ADT. ${ }^{28,30}$ The prevalence of osteoporosis in prostate cancer patients during ADT is 9 to $53 \%{ }^{31}$ In a study of 11,661 patients with nonmetastatic prostate cancer, Smith et al showed a significantly higher fracture rate in men undergoing ADT (7.88 vs. 6.51\%-year of controls, $p<0.001$ ). ${ }^{32}$ Shahinian et al reviewed records of 50,613 men from the Surveillance Epidemiology and End Results and Medicare database reporting a significantly higher proportion of patients who had fractures who received ADT versus who did not (19.4 vs. $12.6 \%, p<0.001$ ); patients receiving ADT experienced a $54 \%$ increase in relative incidence of fractures. ${ }^{20}$

The unadjusted fracture-free survival was the highest in patients who did not receive ADT, whereas it was lowest in patients who underwent orchiectomy followed by ADT with $\geq 9$ doses of $\mathrm{GnRH}$ agonists in the year after diagnosis. ${ }^{20}$

\section{Treatment of Bone Loss in Prostate Cancer}

The European Association of Urology (EAU), International Society of Geriatric Oncology (SIOG), and European Society for Radiotherapy and Oncology (ESTRO) jointly published guidelines for prostate cancer in 2016 recommending dual-energy X-ray absorptiometry (DEXA) assessment for evaluation of BMD in all men starting long-term ADT and assessing the individual risk for fractures. ${ }^{33}$ The repeat measurement of BMD after the first year of ADT is recommended by the International Society for Clinical Densitometry. Lifestyle modification and pharmacologic interventions are potential strategies for the management of bone loss in men with prostate cancer receiving ADT. ${ }^{5}$

\section{Pharmacologic Interventions}

Vitamin D alone or in combination with calcium has been shown to be beneficial with respect to increase in BMD and fall and fracture prevention. ${ }^{34,35}$ Studies have reported the effectiveness of bisphosphonates in men with nonmetastatic prostate cancer receiving ADT. ${ }^{5}$ The advent of denosumab as an osteoprotective agent has significantly improved therapeutic options in prostate cancer. ${ }^{36}$ Zoledronic acid, denosumab, alendronate, and risedronate are the recommended first-line agents for the pharmacologic interventions in men receiving ADT. ${ }^{5}$ Zoledronic acid is one of the most commonly used bone modifying agent. However, treatment with intravenous (IV) zoledronic acid requires hospital visits once every few months (range: 3-12 months) which puts the patients on burden for travel, waiting, set up, and monitoring time, and a 15-minute infusion time. ${ }^{37,38}$ Need for intravenous access and administration, regular requirement of renal function monitoring with necessary dose adjustments, and treatment of renal damage caused in patients during the therapy are some other challenges with zoledronic acid treatment. ${ }^{37,38}$ Denosumab treatment does not have these limitations, given subcutaneously. It does not require renal function monitoring/dose modifications or hospitalization for drug administration. ${ }^{39}$ Denosumab subcutaneous injections (once in every 6 months) can be administered by the patients themselves, though some patients prefer injections through health care professionals. In addition to its efficacy and safety advantages, subcutaneous denosumab demonstrates a higher patient adherence than oral or intravenous bisphosphonates. ${ }^{40}$ Oral bisphosphonates require that patients should not lie down; hence, denosumab can be a good option in patients who are unable to sit or stand..$^{41}$ Denosumab, in addition to improving the BMD, also reduces the incidence of new radiographic vertebral fractures, whereas bisphosphonates increase the BMD but a meta-analysis concluded that evidence was not sufficiently powered to detect fracture reduction. ${ }^{42}$

\section{Denosumab Prevents Bone Loss: Mechanism of Action}

Denosumab binds to RANKL with a high specificity and affinity which inhibits the recruitment and maturation of osteoclast and thus diminishes its action leading to a slowed bone resorption. ${ }^{43}$

\section{Denosumab for Prevention of Bone Loss in Prostate Cancer: What Guidelines Say?}

According to National Comprehensive Cancer Network ( $\mathrm{NCCN}$ ), the treatment options to increase bone density in men without metastases or to increase BMD during ADT for prostate cancer includes denosumab, zoledronic acid, or alendronate. The U.S. Food and Drug Administration has approved (2011) denosumab as a treatment to increase bone mass in nonmetastatic prostate cancer patients with high risk of fracture from receiving ADT. ${ }^{11}$

\section{Guideline Recommendations}

- Fracture risk assessment using free online World Health Organization Fracture Risk Assessment Tool (FRAX). ${ }^{44}$

- The National Osteoporosis Foundation (NOF) guidelines recommend BMD testing in men aged $\geq 70$ years or vertebral imaging in men aged 70 to 79 years if BMD T-score at the spine, total hip, or femoral neck is $\leq-1.5 .^{45}$

- The NCCN recommends treatment with denosumab (60 mg, once in every 6 months), zoledronic acid (5 mg IV once in every 12 months), or alendronate ( $70 \mathrm{mg}$ PO weekly) when absolute fracture risk warrants drug therapy. ${ }^{44}$

- Pretreatment and a follow-up DEXA scan post 1 year of therapy. ${ }^{44}$

- Use of biochemical markers of bone turnover is not recommended. ${ }^{44}$

- The NOF recommends evaluation of laboratory parameters including renal function, magnesium, calcium, vitamin $\mathrm{D}$, phosphorus, and testosterone levels to differentiate the risk of osteopenia or osteoporosis. ${ }^{45}$

- No existing guidelines on the optimal frequency of vitamin D testing, but vitamin D levels can be measured when DEXA scans are obtained. ${ }^{44}$

As per the Institute of Medicine (IOM), recommended dietary allowances for vitamin $\mathrm{D}$ and calcium are $600 \mathrm{IU}$ and $1,000 \mathrm{mg}$ for men aged 51 to 70 years, and $800 \mathrm{IU}$ and $1,200 \mathrm{mg}$ for $>70$ years, respectively. ${ }^{46}$

We review the available evidence of denosumab for managing bone loss in nonmetastatic prostate cancer patients. 
Denosumab for Treatment of Bone Loss in Nonmetastatic Prostate Cancer Hormone Ablation bone Loss Trial

The effects of denosumab on BMD and fractures in men receiving ADT for nonmetastatic prostate cancer were evaluated in a large ( $n=1,468)$, multinational, phase-III Hormone Ablation bone Loss Trial (HALT) by Smith and colleagues. ${ }^{47}$ In this double-blind, placebo-controlled study, patients aged $\geq 70$ years ( $<70$ years with $\mathrm{T}$ score $<-1.0$ or history of osteoporotic fractures) with histologically confirmed prostate cancer were randomized to denosumab ( $n=974,60 \mathrm{mg}$ subcutaneously, once in every 6 months) or placebo $(n=974)$ with daily calcium ( $\geq 1 \mathrm{~g}$ ) and vitamin D ( $\geq 400 \mathrm{IU})$ supplementation. The percentage change in lumbar spine BMD at 24 months was the primary endpoint.

\section{Denosumab and Increase in Bone Mineral Density in Nonmetastatic Prostate Cancer}

Denosumab significantly improved BMD at the lumbar spine from baseline after 24 and 36 months versus placebo (increase in BMD at 24 months: $5.6 \%$ for denosumab vs. $-1 \%$ for placebo; $p<0.001$; absolute difference $6.7 \%$ ) in the HALT study.

The significant ( $p<0.001$ at all the time points) differences in BMD between the two groups were seen as early as 1 month and sustained through 36 months. ${ }^{47}$ Furthermore, denosumab resulted in significant increases in BMD at the total hip, femoral neck, and distal third of the radius during the 36 months. ${ }^{48}$

\section{Denosumab and Decrease in Fractures in Nonmetastatic Prostate Cancer}

There was a $62 \%$ decreased incidence of new vertebral fractures at 36 months with denosumab (relative risk [RR]: 0.38; $95 \%$ confidence interval $[\mathrm{CI}]: 0.19$ to $0.78 ; p=0.006) .{ }^{47}$

Also, there was a lower incidence of fractures at any site with denosumab as compared with placebo at 36 months (5.2 vs. $7.2 \% ; p=0.10$ ). With denosumab, a significantly lesser number of patients developed $\geq 1$ fracture at any site versus placebo ( $0.7 \%$ vs. $2.5 \% ; p=006)$; time to first clinical fracture was not significantly different between the groups. ${ }^{47}$

\section{Denosumab and Decrease in Bone Biomarkers in Nonmetastatic Prostate Cancer}

Denosumab treatment decreased the levels of bone biomarkers, relative to baseline, to a significantly ( $p<0.001$ ) greater extent than placebo (serum C-telopeptide [a biomarker of bone resorption]: $45 \%$ versus $13 \%$; procollagen type I N-terminal peptide [a biomarker of bone formation]: $61 \%$ vs. $18 \%$; and tartrate-resistant acid phosphatase 5b: $33 \%$ vs. $8 \%)^{4}{ }^{4}$

\section{Denosumab versus Other Bone Modifying Agents in Nonmetastatic Prostate Cancer}

Reducing the risk of fractures: A systematic review and network meta-analysis by Poon et al demonstrated that bisphosphonates, denosumab, toremifene, and raloxifene effectively reduced the rate of bone loss when compared with placebo.
Denosumab and zoledronic acid improved BMD across all sites (total hip and femoral neck: $3 \%$ and lumbar spine: $6 \%$ ). The surface under the cumulative ranking curve (SUCRA) determined the treatment with the highest probability of being the most effective in improving BMD and showed that zoledronic acid ranked among the top two treatments at all sites, whereas denosumab was ranked among the top two at the lumbar spine and total hip sites. ${ }^{37}$

Prevention of bone metastases: A systematic review and meta-analysis of six randomized controlled trials (RCTs) by Hayes et al evaluated the effects of zoledronic acid (five studies) and denosumab (one study) on prevention of bone metastasis in men $(n=5,947)$ with high-risk, nonmetastatic prostate cancer. The pooled analysis did not demonstrate any difference in the incidence of bone metastases between zoledronic acid/denosumab treatments versus placebo (RR: $1.09,95 \%$ CI: $0.84-1.41, p=0.51$ ). Only the denosumab study was the positive trial among the included studies (RR: 0.83, $95 \% \mathrm{CI}: 0.73-0.95, p=0.007)$, and this analysis concluded that denosumab delays the onset of bone metastases in high-risk nonmetastatic prostate cancer patients, while there is a limited evidence for zoledronic acid. ${ }^{49}$

\section{Denosumab versus Alendronate: Increase in Bone Mineral Density and Bone Turnover Markers}

Denosumab (60 mg subcutaneous [SC], once in every 6 months) significantly increased the BMD and bone turnover markers with a lower rate of new vertebral fractures as compared with alendronate (weekly $70 \mathrm{mg}$ ) in 234 patients (aged $\geq 55$ years, a $\mathrm{T}$ score of $<-1.0$ and $\geq 1$ fragility fracture) with osteoporosis in a randomized controlled trial by Doria and colleagues. ${ }^{39}$ Denosumab treatment resulted in a significantly improved BMD at lumbar spine at 2 years when compared with alendronate (5.6 vs. $-1.1 \%$; absolute difference: $6.7 \% ; p<0.001)$. A significant difference between the groups was reported for trabecular BMD. ${ }^{39}$ Improvement in back pain from baseline versus all the time points as noted by visual analog scale (VAS) score was significant for both the groups (-Fig. 2). ${ }^{39}$ The study also showed significant improvements in health-related QOL for both treatment groups. ${ }^{39}$

\section{Safety-Denosumab versus Other Agents}

Denosumab is contraindicated in patients with hypercalcemia. Hypocalcemia, osteonecrosis of the jaw (ONJ), clinically significant hypersensitivity reactions, atypical fractures, serious infections, dermatologic reactions, musculoskeletal pain, and bone turnover suppression are the adverse events [AEs] of clinical interest with denosumab. ${ }^{50,51}$ Arthralgia, back pain, pain in extremity, and musculoskeletal pain are the most common AEs reported with denosumab in patients with bone loss receiving ADT for prostate cancer. ${ }^{52}$ Skeletal-related events are cause of significant morbidity in prostate cancer patients. ${ }^{53}$

In the phase-III HALT study in nonmetastatic prostate cancer patients $(n=1,468)$, the rates of AEs were similar for denosumab versus placebo; no cases of ONJ were reported. The most common AEs reported by $\geq 10 \%$ of patients receiving 


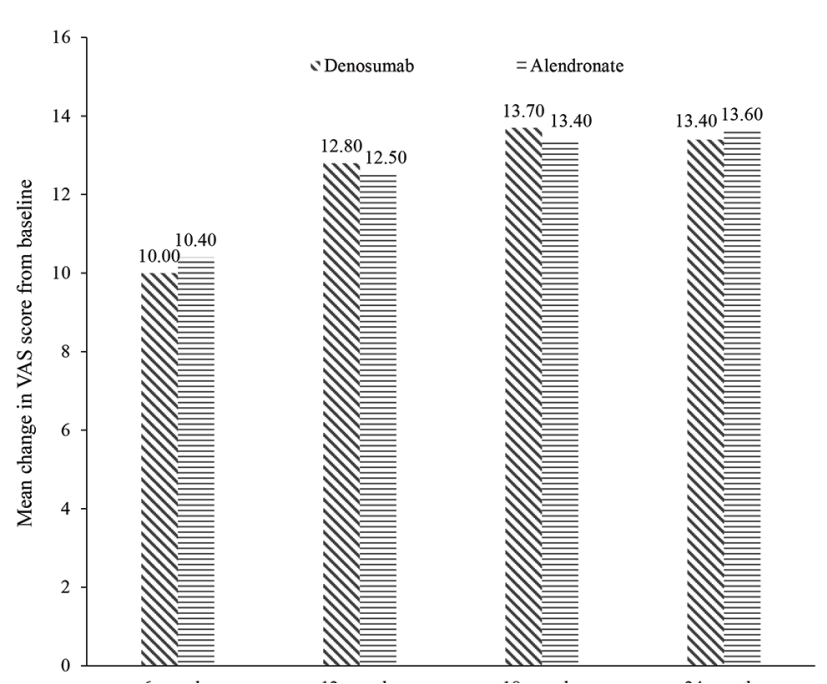

Fig. 2 Improvement in back pain from baseline to all time points in denosumab and alendronate groups. ${ }^{39}$ VAS, visual analog scale.

denosumab were arthralgia, pain in extremity, back pain, fatigue, constipation, cough, and insomnia. ${ }^{47}$ In the study, comparing denosumab versus alendronate in the treatment of osteoporosis secondary to hypogonadism in prostate cancer, denosumab treatment was associated with a higher incidence of hypocalcemia (9.7 vs. $0 \%$ ), abdominal discomfort (9.7 vs. 3.2\%) and decreased appetite (9.7 vs. 3.2\%), whereas alendronate had higher incidence for eczema (6.4 vs. 3.2\%), urticaria (12.9 vs. $3.2 \%)$, rash (9.7 vs. $6.4 \%$ ), and headache (12.9 vs. 3.2\%); nausea was similar (3.2\% in each group). ${ }^{39}$

Bisphosphonate-associated $\mathrm{ONJ}$ is a serious complication with high dose or longer term use of zoledronic acid. ${ }^{7}$ Similarly, ONJ is reported with denosumab use also (overall incidence in cancer patients: $1.7 \%$ [95\% CI: 0.9-3.1\%]). A meta-analysis of 8,963 cancer patients reported a significantly increased risk of ONJ with denosumab treatment when compared with bisphosphonates/placebo (relative risk: 1.61, 95\% CI: 1.05-2.48, $p=0.029) .{ }^{54} \mathrm{~A}$ dental examination with appropriate preventive dentistry is recommended prior to denosumab treatment in high-risk patients. ${ }^{52}$

Hypocalcemia is reported frequently in prostate cancer patients receiving bisphosphonates or denosumab due to the reduced osteoclast activity, causing a decline in the release of calcium from bone. ${ }^{55}$ Hypocalcemia can be worsened in patients with renal impairment or undergoing dialysis with denosumab administration. A regular monitoring of calcium and minerals (magnesium and phosphorus) within 14 days is required in patients receiving denosumab. ${ }^{52}$

Prostate cancer occurs majorly in elderly patients and renal impairment is very common in these patients. ${ }^{39}$ Zoledronic acid is known to cause renal impairment in prostate cancer patients and an elevated serum creatinine level is seen within 15 minutes of administration. ${ }^{56}$ Zoledronic acid clearance occurs through kidneys and dose modifications are required in patients with renal dysfunction. ${ }^{57}$ Denosumab does not alter renal functioning; hence, there is no requirement of renal monitoring or dose modifications in these patients. ${ }^{39}$
The effects of denosumab on bone resorption stops after the discontinuation, as it is not incorporated in the bone, unlike bisphosphonates. ${ }^{58}$ Following discontinuation of denosumab, an increase in the fracture risk, including the risk of multiple vertebral fractures, is reported. A reversal of bone turnover to pretreatment values within 24 months, BMD within 18 months and new fractures within 7 months are reported after denosumab stoppage. Hence, denosumab treatment should not be stopped until a rapid transition to alternative (antiresorptive) treatment is made..$^{58,59}$

\section{Cost Effectiveness of Bisphosphonates versus Denosumab}

Denosumab was found a cost-effective treatment option versus zoledronic acid in a study in the Czech Republic in prostate cancer patients. The incremental cost per quality-adjusted life-years (QALY) gained for denosumab versus zoledronic acid was 382,673 CZK. ${ }^{60}$ Stopeck and colleagues concluded cost effectiveness with denosumab from both payer and societal perspectives in preventing bone complications in patients with solid tumors. ${ }^{61}$ However, cost-effectiveness data on denosumab use in nonmetastatic prostate cancer is not available to our knowledge.

Several biosimilar denosumab formulations are being developed to provide a cost-effective alternative to Prolia (innovator denosumab manufactured by Amgen, United States), of which "Esentra (Intas Pharmaceuticals Limited, Ahmedabad, India)," is the only biosimilar denosumab available to Indian patients. The biosimilar denosumab is approved for the treatment to increase bone mass in men with osteoporosis at high risk of fracture and to increase bone mass in men at high risk for fracture receiving ADT for nonmetastatic prostate cancer.

\section{Conclusion}

The decrease in BMD and increased risk of fractures is of significant concern in patients with prostate cancer who are mostly elderly and are already at a risk of developing osteoporosis. The ADT commonly used for the treatment of prostate cancer that further increases the risk of bone loss in these patients. Bisphosphonates and denosumab are the established agents in the management of the bone loss and fractures in patients with prostate cancer. Denosumab $60 \mathrm{mg}$ SC in every 6 months is approved for the treatment to increase bone mass in men at high risk for fractures receiving ADT for nonmetastatic prostate cancer. Denosumab prevents bone loss, maintains BMD, delays the onset of bone metastasis, and has a good safety profile. Biosimilar denosumab can be a preferred treatment option for the treatment of bone loss in nonmetastatic prostate cancer.

\footnotetext{
Author's Contribution

The manuscript has been developed by the author, the requirements for authorship as stated earlier in this document have been met, and the author believes that the manuscript represents honest work.
} 


\section{Funding}

None.

\section{Conflict of Interest}

None declared.

\section{References}

1 Bray F, Ferlay J, Soerjomataram I, et al. Global cancer statistics 2018: GLOBOCAN estimates of incidence and mortality worldwide for 36 cancers in 185 countries. CA Cancer J Clin 2018;68(6):394-424

2 Palmbos PL, Hussain M. Non-castrate metastatic prostate cancer: have the treatment options changed? Semin Oncol 2013;40(3):337-346

3 Gilbert SM, Kuo YF, Shahinian VB. Prevalent and incident use of androgen deprivation therapy among men with prostate cancer in the United States. Urol Oncol 2011;29(6):647-653

4 Meng MV, Grossfeld GD, Sadetsky N, et al. Contemporary patterns of androgen deprivation therapy use for newly diagnosed prostate cancer. Urology 2002;60(3, suppl 1):7-11, discussion 11-12

5 Lee CE, Leslie WD, Czaykowski P, et al. A comprehensive bone-health management approach for men with prostate cancer receiving androgen deprivation therapy. Curr Oncol 2011;18(4):e163-e172

6 Matsushima $\mathrm{H}$. Prostate cancer and cancer treatment-induced bone loss (CTIBL) [in Japanese]. Clin Calcium 2016;26(12):1039-1045

7 Saad F, Adachi JD, Brown JP, et al. Cancer treatment-induced bone loss in breast and prostate cancer. J Clin Oncol 2008;26(33):5465-5476

8 Maxwell C, Viale $\mathrm{PH}$. Cancer treatment-induced bone loss in patients with breast or prostate cancer. Oncol Nurs Forum 2005;32(3):589-603

9 Charles JF, Aliprantis AO. Osteoclasts: more than 'bone eaters' Trends Mol Med 2014;20(8):449-459

10 BoyceBF,XingL.FunctionsofRANKL/RANK/OPGinbonemodeling and remodeling. Arch Biochem Biophys 2008;473(2):139-146

11 Planas Morin J, Morote Robles J. Skeletal complications of ADT: disease burden and treatment options. Asian J Androl 2012;14(5):670-675

12 Jones DH, Kong Y-Y, Penninger JM. Role of RANKL and RANK in bone loss and arthritis. Ann Rheum Dis 2002;61(suppl, 2): ii32-ii39

13 Gholz RC, Conde F, Rutledge DN. Osteoporosis in men treated with androgen suppression therapy for prostate cancer. Clin J Oncol Nurs 2002;6(5):88-93

14 Leder BZ, LeBlanc KM, Schoenfeld DA, Eastell $R$, Finkelstein JS. Differential effects of androgens and estrogens on bone turnover in normal men. J Clin Endocrinol Metab 2003;88(1):204-210

15 Chen Z, Maricic M, Nguyen P, et al. Low bone density and high percentage of body fat among men who were treated with androgen deprivation therapy for prostate carcinoma. Cancer 2002;95(10):2136-2144

16 James ND, Sydes MR, Clarke NW, et al. Addition of docetaxel, zoledronic acid, or both to first-line long-term hormone therapy in prostate cancer (STAMPEDE): survival results from an adaptive, multiarm, multistage, platform randomised controlled trial. Lancet 2016;387(10024):1163-1177

17 Buehring B, Viswanathan R, Binkley N, Busse W. Glucocorticoid-induced osteoporosis: an update on effects and management. J Allergy Clin Immunol 2013;132(5):1019-1030

18 Handforth C, D'Oronzo S, Coleman R, Brown J. Cancer treatment and bone health. Calcif Tissue Int 2018;102(2):251-264
19 Rachner TD, Coleman R, Hadji P, Hofbauer LC. Bone health during endocrine therapy for cancer. Lancet Diabetes Endocrinol 2018;6(11):901-910

20 Shahinian VB, Kuo YF, Freeman JL, Goodwin JS. Risk of fracture after androgen deprivation for prostate cancer. $\mathrm{N}$ Engl J Med 2005;352(2):154-164

21 Greenspan SL, Coates P, Sereika SM, et al. Bone loss after initiation of androgen deprivation therapy in patients with prostate cancer. J Clin Endocrinol Metab 2005;90(12):6410-6417

22 Morote J, Orsola A, Abascal JM, et al. Bone mineral density changes in patients with prostate cancer during the first 2 years of androgen suppression. J Urol 2006;175(5):1679-1683, discussion 1683

23 Wadhwa VK, Weston R, Mistry R, Parr NJ. Long-term changes in bone mineral density and predicted fracture risk in patients receiving androgen-deprivation therapy for prostate cancer, with stratification of treatment based on presenting values. BJU Int 2009;104(6):800-805

24 Maillefert JF, Sibilia J, Michel F, et al. Bone mineral density in men treated with synthetic gonadotropin-releasing hormone agonists for prostatic carcinoma. J Urol 1999;161(4):1219-1222

25 Gralow JR, Biermann JS, Farooki A, et al. NCCN task force report: bone health in cancer care. J Natl Compr Canc Netw 2013;11(suppl 3):S1-S50, quiz S51

26 Mitsuzuka K, Arai Y. Metabolic changes in patients with prostate cancer during androgen deprivation therapy. Int J Urol 2018;25(1):45-53

27 Oefelein MG, Ricchuiti V, Conrad W, et al. Skeletal fracture associated with androgen suppression induced osteoporosis: the clinical incidence and risk factors for patients with prostate cancer. J Urol 2001;166(5):1724-1728

28 Guise TA, Oefelein MG, Eastham JA, et al. Estrogenic side effects of androgen deprivation therapy. Rev Urol 2007;9(4):163-180

29 Morote J, Morin JP, Orsola A, et al. Prevalence of osteoporosis during long-term androgen deprivation therapy in patients with prostate cancer. Urology 2007;69(3):500-504

$30 \mathrm{Wu}$ CT, Yang YH, Chen PC, Chen MF, Chen WC. Androgen deprivation increases the risk of fracture in prostate cancer patients: a population-based study in Chinese patients. Osteoporos Int 2015;26(9):2281-2290

31 Lassemillante AC, Doi SA, Hooper JD, Prins JB, Wright OR. Prevalence of osteoporosis in prostate cancer survivors: a meta-analysis. Endocrine 2014;45(3):370-381

32 Smith MR, Lee WC, Brandman J, et al. Gonadotropin-releasing hormone agonists and fracture risk: a claims-based cohort study of men with nonmetastatic prostate cancer. J Clin Oncol 2005;23(31):7897-7903

33 Mottet N, Bellmunt J, Bolla M, et al. EAU-ESTRO-SIOG guidelines on prostate cancer. Part 1: screening, diagnosis, and local treatment with curative intent. Eur Urol 2017;71(4):618-629

34 Jackson RD, LaCroix AZ, Gass M, et al. Calcium plus vitamin D supplementation and the risk of fractures. N Engl J Med 2006;354(7):669-683

35 Bischoff-Ferrari HA, Dawson-Hughes B, Willett WC, et al. Effect of vitamin D on falls: a meta-analysis. JAMA 2004;291(16):1999-2006

36 Fizazi K, Carducci M, Smith M, et al. Denosumab versus zoledronic acid for treatment of bone metastases in men with castration-resistant prostate cancer: a randomised, double-blind study. Lancet 2011;377(9768):813-822

37 Poon Y, Pechlivanoglou P, Alibhai SMH, et al. Systematic review and network meta-analysis on the relative efficacy of osteoporotic medications: men with prostate cancer on continuous androgen-deprivation therapy to reduce risk of fragility fractures. BJU Int 2018;121(1):17-28

38 Khan AA, Morrison A, Hanley DA, et al. Diagnosis and management of osteonecrosis of the jaw: a systematic review and international consensus. J Bone Miner Res 2015;30(1):3-23 
39 Doria C, Leali PT, Solla F, et al. Denosumab is really effective in the treatment of osteoporosis secondary to hypogonadism in prostate carcinoma patients? A prospective randomized multicenter international study. Clin Cases Miner Bone Metab 2016;13(3):195-199

40 Hadji P, Kyvernitakis I, Kann PH, et al. GRAND-4: the German retrospective analysis of long-term persistence in women with osteoporosis treated with bisphosphonates or denosumab. Osteoporos Int 2016;27(10):2967-2978

41 Razaq A, Khan S, Hassan J, Malik BH, Razaq M. Comparing the efficacy and safety of denosumab with bisphosphonates in increasing bone mineral density in patients with prostate cancer and breast cancer on antihormonal treatment. Cureus 2019;11(12):e6401-e6401

42 Alibhai SMH, Zukotynski K, Walker-Dilks C, et al. Bone health and bone-targeted therapies for nonmetastatic prostate cancer: a systematic review and meta-analysis. Ann Intern Med 2017;167(5):341-350

43 Boyle WJ, Simonet WS, Lacey DL. Osteoclast differentiation and activation. Nature 2003;423(6937):337-342

44 NCCN Clinical Practice Guidelines in Oncology. NCCN guidelines for patients. Available at: https://www.nccn.org/ patients/guidelines/content/PDF/prostate-advanced-patient. pdf. Accessed June 30, 2021

45 Cosman F, de Beur SJ, LeBoff MS, et al. National Osteoporosis Foundation. Clinician's guide to prevention and treatment of osteoporosis. Osteoporos Int 2014;25(10):2359-2381

46 Ross AC. The 2011 report on dietary reference intakes for calcium and vitamin D. Public Health Nutr 2011;14:938-939

47 Smith MR, Egerdie B, Hernández Toriz N, et al. Denosumab in men receiving androgen-deprivation therapy for prostate cancer. N Engl J Med 2009;361(8):745-755

48 Smith MR, Saad F, Egerdie B, et al. Effects of denosumab on bone mineral density in men receiving androgen deprivation therapy for prostate cancer. J Urol 2009;182(6):2670-2675

49 Hayes AR, Brungs D, Pavlakis N. Osteoclast inhibitors to prevent bone metastases in men with high-risk, non-metastatic prostate cancer: A systematic review and meta-analysis. PLoS One 2018;13(1):e0191455

50 Pond GR, Armstrong AJ, Wood BA, et al. Evaluating the value of number of cycles of docetaxel and prednisone in men with metastatic castration-resistant prostate cancer. Eur Urol 2012;61(2):363-369
51 Foncubierta A, Gutierrez D, Medina F, et al. Hypersensitivity reaction to denosumab in patient with osteoporosis: desensitization methodology. J Allergy Clin Immunol 2014;133 (2, suppl):AB272

52 Prolia (denosumab). Highlights of prescribing information. Available at: https://www.accessdata.fda.gov/drugsatfda docs/label/2020/125320s205lbl.pdf. Accessed July 21, 2020

53 Saad F, Ivanescu C, Phung D, et al. Skeletal-related events significantly impact health-related quality of life in metastatic castration-resistant prostate cancer: data from PREVAIL and AFFIRM trials. Prostate Cancer Prostatic Dis 2017;20(1):110-116

54 Boquete-Castro A, Gómez-Moreno G, Calvo-Guirado JL, Aguilar-Salvatierra A, Delgado-Ruiz RA. Denosumab and osteonecrosis of the jaw. A systematic analysis of events reported in clinical trials. Clin Oral Implants Res 2016;27(3):367-375

55 Body J-J, von Moos R, Niepel D, Tombal B. Hypocalcaemia in patients with prostate cancer treated with a bisphosphonate or denosumab: prevention supports treatment completion. BMC Urol 2018;18(1):81-81

56 Chang JT, Green L, Beitz J. Renal failure with the use of zoledronic acid. N Engl J Med 2003;349(17):1676-1679

57 Stopeck AT, Lipton A, Body JJ, et al. Denosumab compared with zoledronic acid for the treatment of bone metastases in patients with advanced breast cancer: a randomized, double-blind study. J Clin Oncol 2010;28(35):5132-5139

58 Cummings SR, Ferrari S, Eastell R, et al. Vertebral fractures after discontinuation of denosumab: a post hoc analysis of the randomized placebo-controlled FREEDOM trial and its extension. J Bone Miner Res 2018;33(2):190-198

59 Tsourdi E, Langdahl B, Cohen-Solal M, et al. Discontinuation of denosumab therapy for osteoporosis: a systematic review and position statement by ECTS. Bone 2017;105:11-17

60 Cristino J, Finek J, Jandova P, et al. Cost-effectiveness of denosumab versus zoledronic acid for preventing skeletal-related events in the Czech Republic. J Med Econ 2017;20(8):799-812

61 Stopeck A, Brufsky A, Kennedy L, et al. Cost-effectiveness of denosumab for the prevention of skeletal-related events in patients with solid tumors and bone metastases in the United States. J Med Econ 2020;23(1):37-47 